Two- di mensi onal cryst al st ruct ure for med by two components of DNA nanoparticl es on a subst $r$ at $e$

\begin{tabular}{|l|l|}
\hline 著者 & Kat suno Hi r oyasu, Negawa Yuya, Sat o Nasahi de \\
\hline $\begin{array}{l}\text { j our nal or } \\
\text { publ i cat i on ti t l e }\end{array}$ & Journal of the Physi cal Soci ety of Japan \\
\hline vol une & 85 \\
\hline nunber & 7 \\
\hline page r ange & 074605 \\
\hline year & $2016-07-15$ \\
\hline URL & ht t p: //hdl . handl e. net /2297/45903 \\
\hline
\end{tabular}




\title{
Two-dimensional Crystal Structure Formed by Two Components of DNA Nanoparticles on a Substrate
}

\author{
Hiroyasu Katsuno ${ }^{1}$, Yuya Maegawa ${ }^{2}$, and Masahide Sato ${ }^{3}$ \\ ${ }^{1}$ Department of Physical Sciences, Ritsumeikan University, 1-1-1 Noji Higashi, \\ Kusatsu, Shiga 525-8577, Japan \\ ${ }^{2}$ Graduate School of Natural Science and Technology, Kanazawa University, \\ Kakuma-machi, Kanazawa 920-1192, Japan \\ ${ }^{3}$ Information Media Center, Kanazawa University, Kakuma-machi, Kanazawa \\ 920-1192, Japan
}

We study the two-dimensional crystal structure of two components of DNA nanoparticles on a substrate by Brownian dynamics simulation. We use the Lennard-Jones potential as the interaction potential between particles and assume that the interaction length between different types of particles, $\sigma_{\mathrm{AB}}$, is smaller than that between the same types of particles, $\sigma$. Two types of particles form an alloy structure. With decreasing $\sigma_{\mathrm{AB}} / \sigma$, the crystal structure changes from a triangular lattice, to a square lattice, a honeycomb lattice, a rectangular lattice, and a triangular lattice.

\section{Introduction}

Many researchers have paid much attention to the fabrication of various lattice structures since the physical properties of materials depend on their structures. Using nanosize building blocks is one of the methods of fabricating a well-ordered lattice structure. For example, in the research field of electronic properties, Han et al. have shown the use of a high-quality monolayer of closed-packed gold nanoparticles formed by conformal printing as a microcontact-printed charge-trapping layer for applications in flash memories. 'As the obtained devices show good endurance properties and mechanical stabilities, the lattice structure is one of the important key factors for properties of electronic devices. In the field of optical properties, a colloidal crystal with the facecentered cubic lattice is one of the candidates of a three-dimensional photonic crystal. The close-packed crystal can be used as a template for inverse opals with perfect photonic band gaps. ${ }^{?, ?}$ It is necessary to create a large colloidal crystal without defects. 
However, it is difficult to predict and control lattice structures over entropic effects.

Recently, nanoparticles covered with DNA have been investigated as useful building blocks. ?,?,? The size of nanoparticles is on the order of $10 \mathrm{~nm}$, which is larger than atoms. The individual nanoparticles become building blocks on the lattice structure. As the nanoparticles are covered with DNA, the metallic property of the nanoparticles disappears, and the hybridization of DNA is important for the interaction between nanoparticles. The DNA strands covering nanoparticles are programmable and the DNA is a kind of tool for direct particle assembly because DNA double helix formation provides the bridge based on those sequences. By this technique, Nykypanchuk et al. have made the body-centered cubic lattice. ${ }^{?}$ Macfarlane et al. have shown that various three-dimensional superlattices can be fabricated. ? Using a single type of DNA nanoparticle, they have obtained simple structures, namely, face-centered cubic lattice, body-centered cubic lattice, and hexagonal closed-packed structure. Using two types of nanoparticles, they have also obtained some stable superlattices. Isogai et al. have observed the triangular lattice? and the square lattice? of DNA functionalized gold nanoparticles in a water solution by atomic force microscope. These lattices are fabricated on the lipid bilayer substrate. The key points of the lipid bilayer are a large affinity with DNA strands and a large fluidity. When DNA nanoparticles are captured on the lipid substrate, they cannot be desorbed into a water solution, and can diffuse on the substrate easily. Although they have shown the explicit alignment of DNA sequences and obtained lattice structures, the requirement of DNA strands for fabricating any lattice structure is unclear.

In this paper, keeping the formation of the lattice on a substrate using two types of DNA covered gold nanoparticles in mind, we carry out Brownian dynamics simulation and investigate the possibility of the formation of various two-dimensional lattices. We show that the lattice structure depends on the optimal distance in the interaction potential between different types of particles. In Sect. ??, we introduce the model. In Sect. ??, we show results and carry out brief discussions. In Sect. ??, we summarize our results.

\section{Model}

In Ref. ?, it is considered that DNA nanoparticles are strongly bound to a lipid with DNA because of their affinities to each other. Thus, after the two-dimensional migration of the DNA nanoparticles owing to the fluidity of the lipid bilayer, the DNA 
nanoparticles mainly crystallize on the lipid bilayer in an aqueous solution.

In order to investigate the lattice structure formed by DNA nanoparticles on the lipid bilayer, we carry out a Brownian dynamics simulation, which is one of the powerful methods of studying the properties of many-particle systems. Since the DNA nanoparticles receive a random force from water molecules, the equation of motion of the $i$ th particle is given by the Langevin equation:

$$
m \frac{d^{2} \boldsymbol{r}_{i}}{d t^{2}}=-\xi \frac{d \boldsymbol{r}_{i}}{d t}+\boldsymbol{F}_{i}+\boldsymbol{F}_{i}^{\mathrm{B}}
$$

where $m$ is the mass of a particle, $\boldsymbol{r}_{i}$ is the position of the $i$ th particle, $\xi$ is the frictional coefficient, and $\boldsymbol{F}_{i}^{\mathrm{B}}$ is the random force satisfying $\left\langle\boldsymbol{F}_{i}^{\mathrm{B}} \cdot \boldsymbol{F}_{j}^{\mathrm{B}}\right\rangle=4 \xi k_{\mathrm{B}} T \delta_{i j} \delta\left(t-t^{\prime}\right)$. The sum of the interaction force from other particles, $\boldsymbol{F}_{i}$, is given by the gradient of the central force potential $U\left(r_{i j}\right)$ as

$$
\boldsymbol{F}_{i}=\sum_{j} \boldsymbol{F}_{i j}=-\sum_{j \neq i} \frac{\partial U\left(r_{i j}\right)}{\partial r_{i j}} \frac{\boldsymbol{r}_{i j}}{r_{i j}},
$$

where $r_{i j}=\left|\boldsymbol{r}_{i j}\right|=\left|\boldsymbol{r}_{i}-\boldsymbol{r}_{j}\right|$.

The form of the interaction potential $U\left(r_{i j}\right)$ is important. The excluded volume effect is not neglected since the size of the nanoparticles is finite. The short-range repulsive force is required in the interaction potential. On the other hand, synthesis between the DNA strands contributes to an attractive force. ${ }^{?}$ The range of the attraction force is finite because the length of DNA strands is finite. The Lennard-Jones (LJ) potential satisfies those fundamental features except for the long-range attraction. This potential is introduced as the potential between rare gas atoms and also applied to binary systems in the field of glass systems. ${ }^{?}$ For simplicity, we adopt the LJ potential as the interaction potential between DNA nanoparticles.

The interaction potential between the $i$ th and $j$ th particles, $U\left(r_{i j}\right)$, is given by

$$
U\left(r_{i j}\right)=4 \varepsilon_{i j}\left\{\left(\frac{\sigma_{i j}}{r_{i j}}\right)^{12}-\left(\frac{\sigma_{i j}}{r_{i j}}\right)^{6}\right\},
$$

where $\sigma_{i j}$ is the distance where $U\left(\sigma_{i j}\right)=0$. The potential profile is determined by the two parameters, $\varepsilon_{i j}$ and $\sigma_{i j}$. The potential takes its minimum $-\varepsilon_{i j}$ at $r_{i j}=2^{1 / 6} \sigma_{i j}$ : the interaction force is repulsive when $r_{i j}<2^{1 / 6} \sigma_{i j}$ and attractive when $r_{i j}>2^{1 / 6} \sigma_{i j}$. $\varepsilon_{i j}$ represents the strength of an attractive force and $\sigma_{i j}$ is proportional to the optimal distance between particles.

In experiments, ?, two types of nanoparticles covered by DNA are utilized for forming various crystal structures. Taking account of such an experimental situation, we 
consider two types of particles, A and B, in our simulation. The set of the two parameters $\left(\varepsilon_{i j}, \sigma_{i j}\right)$ is classified into three types: $\left(\varepsilon_{\mathrm{AA}}, \sigma_{\mathrm{AA}}\right)$ for the interaction between two A particles, $\left(\varepsilon_{\mathrm{BB}}, \sigma_{\mathrm{BB}}\right)$ for the interaction between two B particles, and $\left(\varepsilon_{\mathrm{AB}}, \sigma_{\mathrm{AB}}\right)$ for the interaction between an A particle and a B particle. Since the origin of the interaction between nanoparticles is the DNA attached to each particle, these variables are related to the designs of DNA strands, namely, the number of amino acid pairs, the number of DNAs attached to the nanoparticle, and so forth. The number of amino acid pairs contributing to the hybridization reflects the magnitude of the potential depth. The optimal distance between nanoparticles is proportional to the length of DNA strands attached to each nanoparticle. Therefore, the magnitude of those parameters in potentials is almost arbitrary because amino acid sequences can be controlled freely. Hereafter, we focus on the optimal distance between different types of nanoparticles. The parameter set is assumed as $\sigma_{\mathrm{AA}}=\sigma_{\mathrm{BB}}=\sigma>\sigma_{\mathrm{AB}}$ and $\varepsilon_{\mathrm{AA}}=\varepsilon_{\mathrm{BB}}=\varepsilon_{\mathrm{AB}}=\varepsilon$.

Since the friction from a solution is very large in the experiment, ${ }^{\text {we neglect the }}$ acceleration in Eq. (??). The velocity of the $i$ th particle is given by

$$
\frac{d \boldsymbol{r}_{i}}{d t}=\frac{\boldsymbol{F}_{i}+\boldsymbol{F}_{i}^{\mathrm{B}}}{\xi} \text {. }
$$

Equation (??) is discretized as?

$$
\tilde{\boldsymbol{r}}_{i}\left(\tilde{t}_{n+1}\right)=\tilde{\boldsymbol{r}}_{i}\left(\tilde{t}_{n}\right)+\left(\tilde{\boldsymbol{F}}_{i}+\tilde{\boldsymbol{F}}_{i}^{\mathrm{B}}\right) \Delta \tilde{t}
$$

where $\tilde{\boldsymbol{r}}_{i}=\boldsymbol{r}_{i} / \sigma, \tilde{t}_{n}=\varepsilon t_{n} / \xi \sigma^{2}, \tilde{\boldsymbol{F}}_{i}=\sigma \boldsymbol{F}_{i} / \varepsilon$, and $\tilde{t}_{n+1}=\tilde{t}_{n}+\Delta \tilde{t}$. The random force $\tilde{\boldsymbol{F}}_{i}^{\mathrm{B}}(\tilde{t})$ satisfies $\left\langle\tilde{\boldsymbol{F}}_{i}^{\mathrm{B}}\left(\tilde{t}_{n}\right) \cdot \tilde{\boldsymbol{F}}_{j}^{\mathrm{B}}\left(\tilde{t}_{m}\right)\right\rangle=4 k_{\mathrm{B}} T \delta_{i j} \delta_{m n} / \varepsilon \Delta \tilde{t}$.

The number of $\mathrm{A}$ and $\mathrm{B}$ particles is 256 each. Thus, the total number of particles is 512 . The system size is $L^{2}=23.73^{2}$ with the periodic boundary condition. The area fraction is $512 \times \pi(\sigma / 2)^{2} / L^{2}=0.9$. The magnitude of the random noise is $k_{B} T / \varepsilon=0.1$.

\section{Results and Discussion}

To investigate the two-dimensional lattice structure for various ratios of $\sigma_{\mathrm{AB}}$ to $\sigma$, we introduce the length parameter $s=\sigma_{\mathrm{AB}} / \sigma$. A very small $s$ seems to be unrealistic because of the steric hindrance. Although the realistic parameter range is unclear, the parameter is set to $0.2<s<1$ for the general study of the effect of $s$ on the lattice structure. Figure ?? shows typical snapshots of lattice structures, where $s$ is (a) 1 , (b) 0.7, (c) 0.6, (d) 0.45, and (e) 0.2. Red open circles and blue filled circles represent the $\mathrm{A}$ and $\mathrm{B}$ particles, respectively. The superficial particle radius is set to about $0.1 \sigma$ in Fig. ??. When $s=1$ [Fig. ??(a)], the connection distance between the A and B particles 

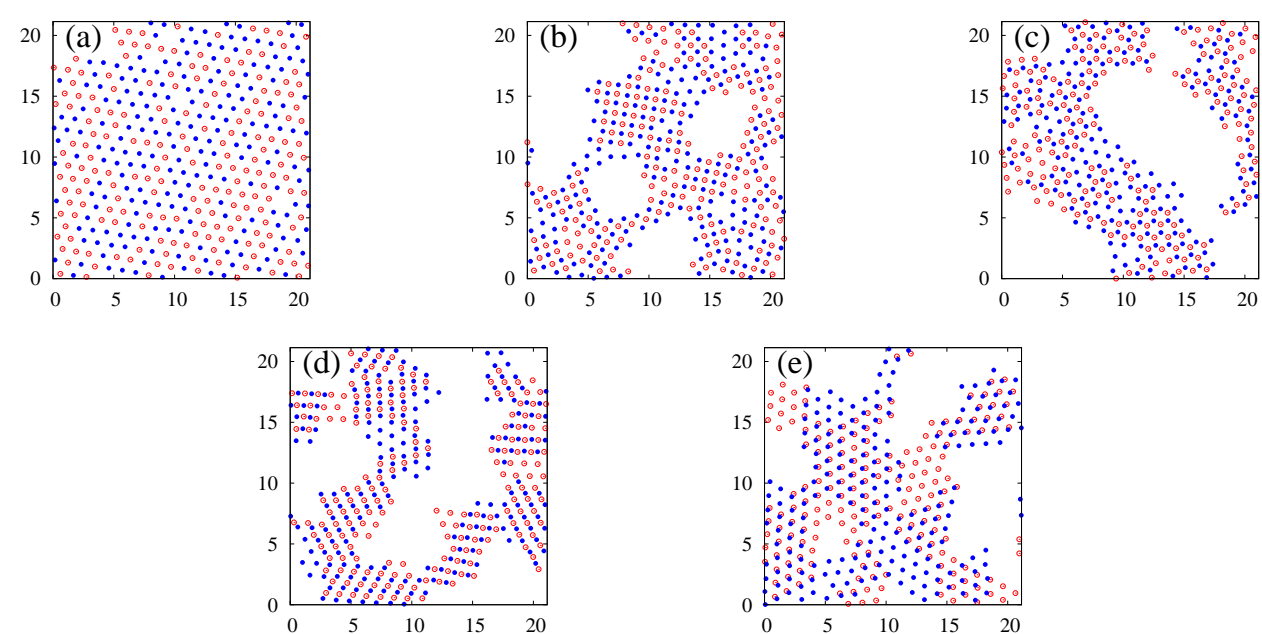

Fig. 1. (Color online) Typical snapshots of lattice structures for (a) $s=1.0$, (b) $s=0.7$, (c) $s=0.6$, (d) $s=0.45$, and (e) $s=0.2$.

is equivalent to that between the same type of particles. Thus, the two types of particles mix at random and a triangular lattice, which is the close-packed structure, appears. With decreasing $s$, the lattice symmetry changes: the square lattice, the hexagonal lattice, and the rectangular lattice are formed when $s$ is $0.7,0.6$, and 0.45 , respectively as shown in Figs. ??(b)-??(d). When $s=0.2$ [Fig. ??(e)], the triangular lattice whose unit is a dimer formed by a combination of the A and B particles seems to emerge. From Fig. ??, we note as follows. The local symmetry of the lattice structure for $s=0.7$ is a square and seems to be different from that for other length parameters. For $s<0.6$ as shown in Figs. ??(c)-??(e), the lattice for the same type of particles seems to be maintained as a triangular although the lattice for all particles changes from a triangular to the other lattices.

To study the relationship between $s$ and the lattice structures more quantitatively, we introduce an order parameter $\phi_{k}(i)$, which shows the local $k$-fold rotational symmetry around the $i$ th particle, and a radial distribution function $\bar{g}(r)$, which shows the characteristic distance between particles. The definition of $\phi_{k}(i)$ is given by

$$
\phi_{k}(i)=\left\{\begin{array}{cc}
\frac{1}{n_{N N}}\left|\sum_{j}^{r<r^{\prime}} e^{\mathrm{i} k \theta_{i j}}\right| & \left(n_{N N} \geq 2\right), \\
0 & \left(n_{N N} \leq 1\right),
\end{array}\right.
$$

where $n_{N N}$ is the number of neighboring particles within the distance $r^{\prime}=1.2 s$, and $\theta_{i j}$ is the angle representing the direction of the $j$ th particle when viewed from the 

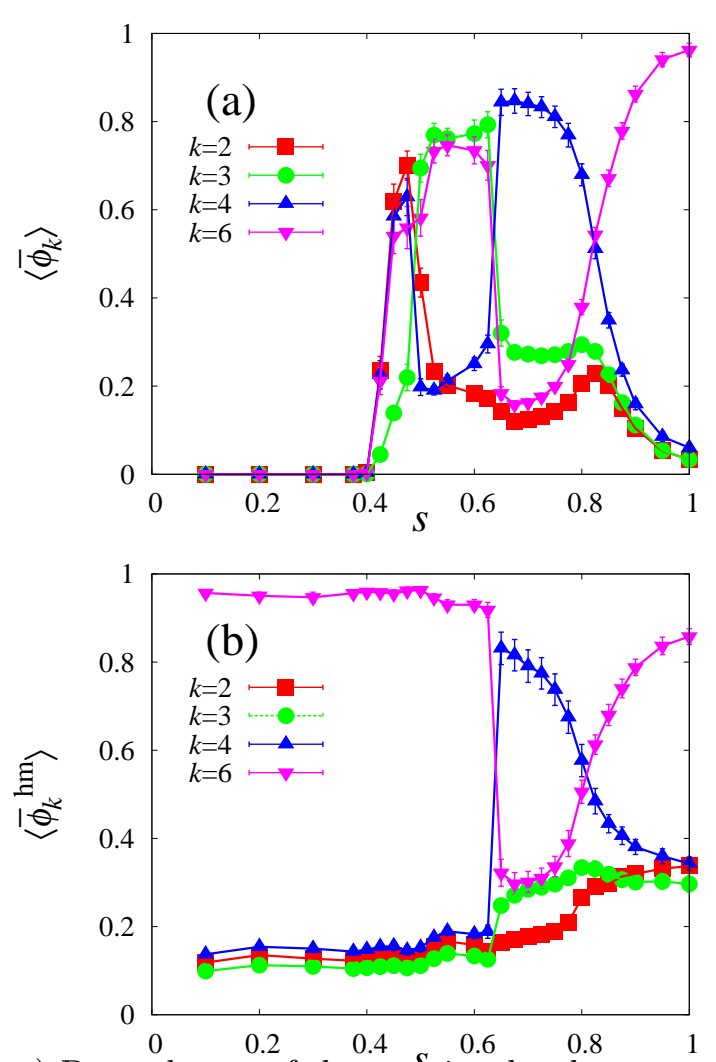

Fig. 2. (Color online) Dependences of the rotational order parameters, (a) $\left\langle\bar{\phi}_{k}\right\rangle$ and (b) $\left\langle\bar{\phi}_{k}^{\mathrm{hm}}\right\rangle$, on $s$. The order parameters are averaged over 100 samples. An error bar represents standard error. Lines are guides for the eyes.

$i$ th particle. Since $s<1, \phi_{k}(i)$ shows the local rotational symmetry of the location of particles whose type is different from that of the $i$ th particle. We also estimate $\phi_{k}^{\mathrm{hm}}(i)$, which shows the rotational symmetry of the particles whose type is the same as that of the $i$ th particle. The definition of $\phi_{k}^{\mathrm{hm}}(i)$ is similar to Eq. (??) except that we only take account of the same type of particles within $r^{\prime}=2^{1 / 6}$. To estimate the $k$-fold rotational symmetry in a system, we use $\bar{\phi}_{k}$ and $\bar{\phi}_{k}^{\mathrm{hm}}$, which are averaged values of $\phi_{k}(i)$ and $\phi_{k}^{\mathrm{hm}}(i)$ in a system, respectively. Moreover, the sample average is taken and the rotational order parameter is denoted by $\left\langle\bar{\phi}_{k}\right\rangle$ and $\left\langle\bar{\phi}_{k}^{\mathrm{hm}}\right\rangle$. We also calculate the radial distribution function $\langle\bar{g}(r)\rangle$ for estimating the ordering of the system. The definition of $\bar{g}(r)$ in a system is given by

$$
\bar{g}(r)=\frac{1}{N} \sum_{i} \frac{n_{i}(r)}{2 \pi r \delta r},
$$

where $n_{i}(r)$ is the number of particles between the distance $r$ to $r+\delta r$ for the $i$ th particle with $\delta r=10^{-2}$. The bracket means the sample average mentioned above. We 
also calculate the radial distribution function for the same type of particles as well in the case of the order parameter.

Figure ?? shows the dependences of the rotational order parameters $\left\langle\bar{\phi}_{k}\right\rangle$ and $\left\langle\bar{\phi}_{k}^{\mathrm{hm}}\right\rangle$ on $s$. Squares, circles, triangles, and inverted triangles represent the dependences of order parameters with $k=2,3,4$, and 6, respectively. From Fig. ??, the rotational lattice symmetry in the range $s<0.4$ seems to be the same as that shown in Fig. ??(e). This parameter region would not be feasible because of its smallness. To study a feasible lattice structure, the radial distribution functions for various $s>0.45$ are shown in Fig. ??. The abscissa represents the normalized length $r / r_{0}$, where $r_{0}$ represents the length of the first nearest neighbor. The value of $s$ is 1 in Figs. ??(a) and ??(b), 0.7 in Figs. ??(c) and ??(d), 0.6 in Figs. ??(e) and ??(f), and 0.45 in Figs. ??(g) and ??(h). The radial distribution functions for all particles are shown in Figs. ??(a), ??(c), ??(e), and ??(g), and for the same type of particles in Figs. ??(b), ??(d), ??(f), and ??(h).
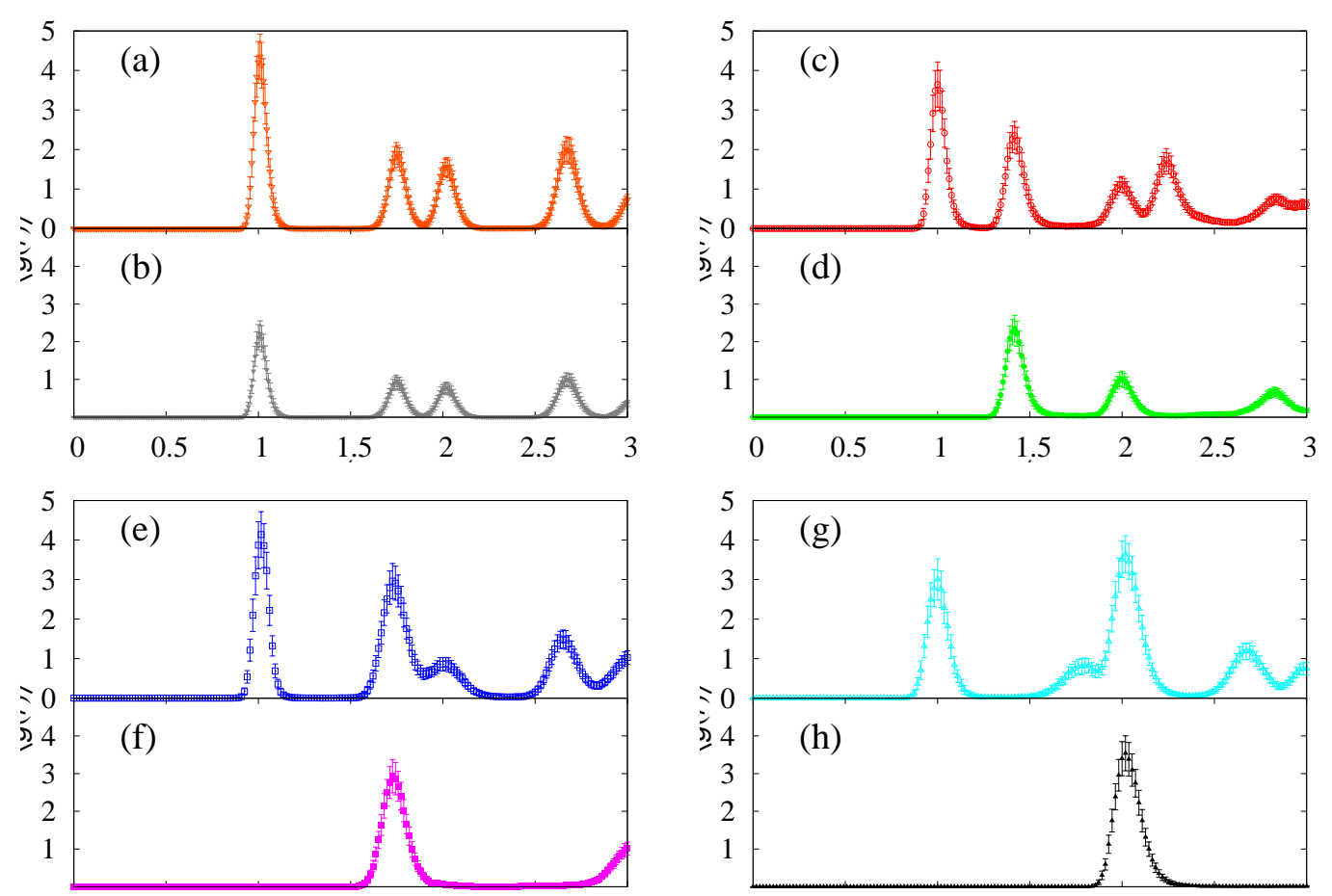

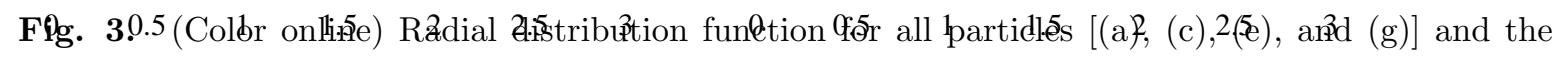
same type of particles [(b), (d), (f), and (h)]. The value of $s$ is 1 in (a) and (b), 0.7 in (c) and (d), 0.6 in (e) and (f), and $0.45 \mathrm{in}(\mathrm{g})$ and (h). The abscissa is rescaled by $r_{0}$ to adjust the position of the first peak to be $1 . r_{0}$ is 1.11 for $s=1,0.79$ for $s=0.7,0.66$ for $s=0.6$, and 0.55 for $s=0.45$. The data are averaged over 100 samples. An error bar represents the standard error. 
When $0.8<s \leq 1$, the large rotational order parameters are $\left\langle\bar{\phi}_{6}\right\rangle$ and $\left\langle\bar{\phi}_{6}^{\mathrm{hm}}\right\rangle$. The system has a sixfold rotational symmetry. The distribution function for the case of $s=1$ [Fig. ??(a)] shows the number of first neighboring particles of about six, although the value of the maximum peak is less than six because of the peak width. In each peak, the ratio of the same type of particles is about half as shown in Figs. ??(a) and ??(b). The triangular lattice with the mixture of $\mathrm{A}$ and $\mathrm{B}$ particles is found as shown in Fig. ??(a) when the difference between $\sigma$ and $\sigma_{\mathrm{AB}}$ is small.

With decreasing $s,\left\langle\bar{\phi}_{6}\right\rangle$ and $\left\langle\bar{\phi}_{6}^{\mathrm{hm}}\right\rangle$ reduce and $\left\langle\bar{\phi}_{4}\right\rangle$ and $\left\langle\bar{\phi}_{4}^{\mathrm{hm}}\right\rangle$ grow gradually. When $0.625<s<0.8,\left\langle\bar{\phi}_{4}\right\rangle$ and $\left\langle\bar{\phi}_{4}^{\mathrm{hm}}\right\rangle$ are larger than other parameters, which means that both A and B particles are located with the fourfold symmetry around a particle. To determine the locations of the particles more clearly, we investigate the radial distribution functions for $s=0.7$ shown in Figs. ??(c) and ??(d). From Fig. ??(c), the positions of the second, third, fourth, and fifth nearest neighbors are estimated as $\sqrt{2} r_{0}, 2 r_{0}, \sqrt{5} r_{0}$, and $2 \sqrt{2} r_{0}$, respectively, and peaks also appear at $\sqrt{2} r_{0}, 2 r_{0}$, and $2 \sqrt{2} r_{0}$ in Fig. ??(d). From the order parameters of the rotational symmetry and the distribution functions, the typical lattice structure is determined as Fig. ??(a), which is consistent with the square lattice observed in Fig. ??(b).

When $s=0.625,\left\langle\bar{\phi}_{k}\right\rangle$ and $\left\langle\bar{\phi}_{k}^{\mathrm{hm}}\right\rangle$ markedly change. Since $\left\langle\bar{\phi}_{4}\right\rangle$ and $\left\langle\bar{\phi}_{4}^{\mathrm{hm}}\right\rangle$ decrease, the square lattices are broken. When $0.475<s<0.625$, large order parameters are $\left\langle\bar{\phi}_{6}\right\rangle,\left\langle\bar{\phi}_{6}^{\mathrm{hm}}\right\rangle$, and $\left\langle\phi_{3}\right\rangle$. When we focus on an A particle, the location of other A particles around the focused A particle has a sixfold rotational symmetry, and B particles are located at least with a threefold rotational symmetry. In Figs. ??(e) and ??(f), we show the radial distribution functions with $s=0.6$. From Fig. ??(e), the positions of the second, third, and fourth nearest neighbors are estimated as $\sqrt{2} r_{0}, \sqrt{3} r_{0}$, and $\sqrt{7} r_{0}$, respectively. The same type of particles appear at the second nearest neighbors as shown in Fig. ??(f). Thus, the expected structure is given by Fig. ??(b), and the honeycomb lattice shown in Fig. ??(c) is formed.

When $0.4<s<0.475,\left\langle\bar{\phi}_{6}^{\mathrm{hm}}\right\rangle$ is large in Fig. ??(b), so that both A and B particles compose the triangular lattice. $\left\langle\bar{\phi}_{2}\right\rangle,\left\langle\bar{\phi}_{4}\right\rangle$, and $\left\langle\bar{\phi}_{6}\right\rangle$ have large values as shown in Fig. ??(a). Around one type of a given particle, another type of particles are located at least with twofold rotational symmetry. The radial distribution functions with $s=0.45$ [Figs. ??(g) and ??(h)] show that the third nearest neighbor located at $\sqrt{3} r_{0}$ consists of the same type of particles. Thus, the typical structure is expected as Fig. ??(c), which is consistent with the rectangular lattice seen in Fig. ??(d). 
(a)
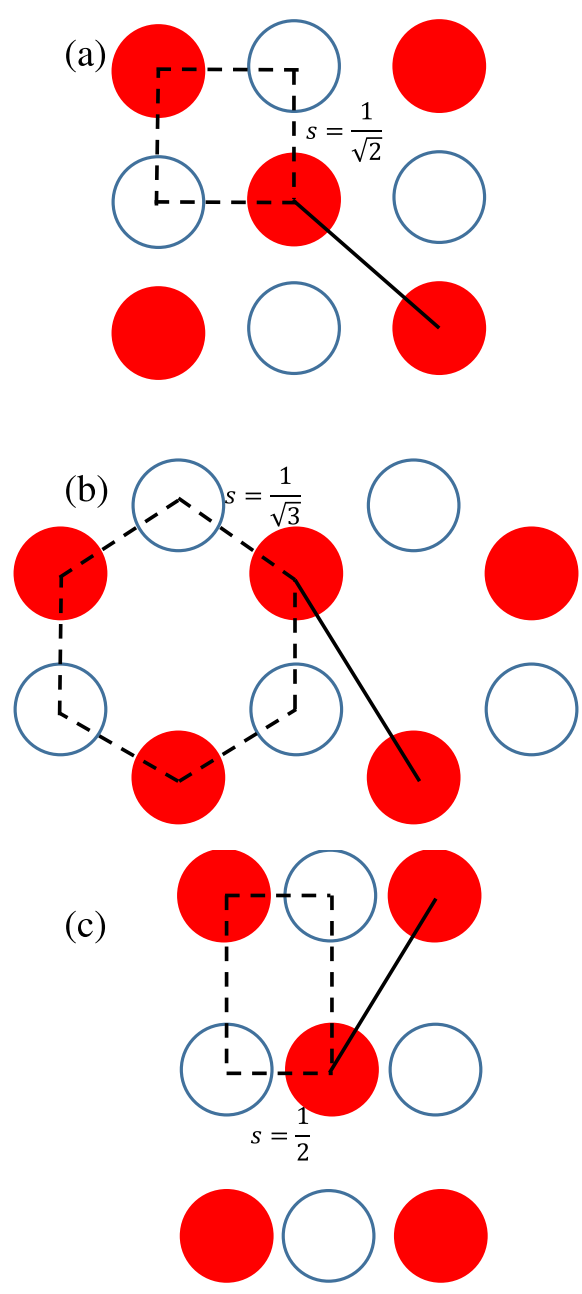

Fig. 4. (Color online) Typical lattice structures and the DNA length parameter $s$ : (a) the square lattice with $s=1 / \sqrt{2}$, (b) the honeycomb lattice with $s=1 / \sqrt{3}$, and (c) the rectangular lattice with $s=1 / 2$. The lengths of the solid line and dashed line show unity and $s$, respectively.

When $s<0.4$, the clear local rotational order does not appear in $\left\langle\bar{\phi}_{k}\right\rangle$ although $\left\langle\phi_{6}^{\mathrm{hm}}\right\rangle$ is larger than 0.9. Thus, the A and B particles form the triangular lattices individually. When triangular lattices are overlapped, dimers of A and B particles are formed and the triangular lattice with the dimers is formed as shown in Fig. ??(e).

To understand the relationship between the parameter $s$ and the lattice structures precisely, we need to consider the dependence of the free energies of the formation of the lattice structures on $s$ carefully. However, when we take into account the interaction between particles up to the second nearest neighbors, we can instinctively understand why the lattice structure changes with the parameter $s$. When the distance between the same type of particles is unity, the distance between the different particles, which 
is given by the parameter $s$, becomes $1 / \sqrt{2} \simeq 0.71$ for a square lattice. Thus, when $0.625<s<0.8$, the A and B particles can form the square lattice stably. In Fig. ??(b), the parameter $s$ becomes $1 / \sqrt{3} \simeq 0.58$, so that the particles are located comfortably with the honeycomb lattice when $0.475<s<0.625$. When the rectangular lattice as shown in Fig. ??(c) is formed, the parameter $s$ is 0.5 , which is consistent with the formation of the rectangular lattice with $0.475<s<0.625$.

\section{Summary}

We demonstrated the lattice structure of the binary system of DNA nanoparticles on the substrate by Brownian dynamics. We focused on the fact that the DNA strands are programmable although the quantitative estimation of the interaction between DNA nanoparticles is difficult. The length of DNA strands and the number of amino acid pairs are probably related to the length of the optimal distance between particles and the depth of the interaction potential well, respectively. The Lennard--Jones potential is applied to the interaction potential between DNA nanoparticles. Two types of particles are introduced and we took into account the difference in length between the different types of particles for our focused features.

With the small length parameter $s<0.4$, the dimer pair is formed and the two types of triangular lattices overlap. When the length parameter increases, the lattice structure changes to the rectangular, honeycomb, square, or triangular lattice. Since the condition of the constant volume and the constant number of particles is used, the lattice density changes for each length parameter. However, the lattice seems to be formed robustly as shown in the rotational symmetry and radial distribution function.

Our results suggest that various lattice structures of nanoparticles can be fabricated since it is possible that the length can be changed by changing a part of the strands unrelated with binding amino acid pairs. We hope that the requirement for fabricating various lattice structures is elucidated through such experiments.

\section{Acknowledgments}

This work is supported by JSPS KAKENHI Grant Numbers 26103515, 26390054, and 16K05470. We benefited from the Joint Research Program of the Institute of Low Temperature Science, Hokkaido University. 


\section{References}

1) S.-T. Han, Y. Zhou, Z.-X. Xu, L.-B. Huang, X.-B. Yang, and V. A. L. Roy, Adv. Mater. 24, 3556 (2011).

2) A. Blanco, E. Chomski, S. Grabtchak, M. Ibisate, S. John, S. W. Leonard, C. Lopez, F. Meseguer, H. Miguez, J. P. Mondia, G. A. Ozin, O. Toader, and H. M. van Drie, Nature 405, 437 (2000).

3) L. Thylen, M. Qiu, and S. Anand, Chem. Phys. Chem. 5, 1268 (2004).

4) C. A. Mirkin, R. L. Letsinger, R. C. Mucic, and J. J. Storhoff, Nature 382, 607 (1996).

5) A. P. Alivisatos, K. P. Johnsson, X. Peng, T. E. Wilson, C. J. Loweth, M. P. Bruchez Jr., and P. G. Schultz, Nature 382, 609 (1996).

6) J. C. Crocker, Nature 451, 528 (2008).

7) D. Nykypanchuk, M. M. Maye, D. van der Lelie, and O. Gang, Nature 451, 549 (2008).

8) R. J. Macfarlane, B. Lee, M. R. Jones, N. Harris, G. C. Schatz, and C. A. Mirkin, Science 334, 204 (2011).

9) T. Isogai, A. Piednoir, E. Akada, Y. Akahosi, R. Tero, S. Harada, T. Ujihara, and M. Tagawa, J. Cryst. Growth 401, 494 (2014).

10) T. Isogai, E. Akada, S. Nakada, N. Yoshida, R. Tero, S. Harada, T. Ujihara, and M. Tagawa, Jpn. J. Appl. Phys. 55, 03DF11 (2016).

11) W. B. Rogers, and J. C. Crocker, Proc. Natl. Acad. Sci. U. S. A. 108, 15687 (2011).

12) W. Kob, and H. C. Andersen, Phys. Rev. Lett. 73, 1376 (1994).

13) D. L. Ermak, J. Chem. Phys. 62, 4189 (1975). 